\title{
Inclusión del emprendimiento en el desarrollo de asignaturas de Ingeniería
}

\section{Introducing entrepreneurship in engineering project management engineering}

\author{
María Dolores Redel-Macías ${ }^{1}$, Carlos Castillo-Rodríguez ${ }^{1}$, Rafael Pérez- \\ Alcántara $^{1}$, Alfonso García-Ferrer ${ }^{2}$, María Santos Hinojosa-Martínez ${ }^{3}$, María \\ Salud Villalba-Evans ${ }^{4}$, Jesús Paniagua-Merchan ${ }^{5}$, Antonio José Cubero- \\ Atienza ${ }^{1}$
}

Fecha de recepción: 25/05/2019; Fecha de revisión: 13/07/2019; Fecha de aceptación: 03/09/2019

Cómo citar este artículo:

Redel-Macías, M.D., Castillo-Rodríguez, C., Pérez-Alcántara, R., García-Ferrer, A., Hinojosa-Martínez, M.S., Villalba-Evans, S., Paniagua-Merchan, J., Cubero-Atienza, A.J. (2019). Inclusión del emprendimiento en el desarrollo de asignaturas de Ingeniería. Revista de Innovación y Buenas Prácticas Docentes, 8(3), 28-42.

Autor de Correspondencia: mdredel@uco.es

\section{Resumen:}

Según el diario el Mundo, por primera vez los estudiantes universitarios españoles que tienen pensado crear un negocio (26,8\%) supera a los que se ven trabajando en la Administración Pública el (25\%), ese fue el resultados de las 8600 encuestas realizadas por GAD3 para el estudio encargado por Educa 2020, donde Ingeniería y Arquitectura son las ramas con mayores porcentajes de alumnado que quieren crear una empresa. Con estos datos, se pretende trabajar en esta línea para estimular al alumnado en la generación de ideas de negocio y dar soporte para llevarlas a cabo. Además, a través del trabajo coordinado con el Vicerrectorado de Coordinación Institucional e Infraestructuras se intentará acercar al alumnado cómo es el proceso de licitación de obras, servicios o equipamiento en una Administración Pública como es la UCO, mostrando la transparencia del proceso. En la experiencia piloto del anterior curso académico, el alumnado valoró muy positivamente la asistencia a un acto público de apertura de ofertas económicas de un proceso de licitación real desarrollado por la UCO, donde se mostraron participativos y vieron de primera mano cómo todo el proceso es transparente y sigue rigurosamente la Ley de Contratos del Sector Público.

Palabras clave: educación en ingeniería, emprendimiento, proyectos de ingeniería.

1 Universidad de Córdoba (España), mdredel@uco.es; ccastillo@uco.es; ir1pealr@uco.es; ir1cuata@uco.es; CÓDIGO ORCID: 0000-0001-5135-9089

2 Universidad de Córdoba (España), ir1gapoa@uco.es

3 Universidad de Córdoba (España), sc1himam@uco.es

4 Universidad de Córdoba (España)

${ }_{5}^{5}$ Asociación de Ingenieros Industriales de Andalucía Occidental (España), delegadocor@coiiaoc.com 


\section{Abstract:}

According to the El Mundo newspaper, for the first time, Spanish university students who plan to create a business (26.8\%) exceed those who are working in the Public Administration (25\%), that was the result of the 8600 surveys conducted by GAD3 for the study commissioned by Educa 2020, where Engineering and Architecture are the branches with the highest percentages of students that want to start a business. With these data, it is recommended to work in this line to stimulate the student in the generation of business ideas and give support to carry them out. In addition, through coordinated work with the Office of the Vice Chancellor for Institutional Coordination and Infrastructures, students are approached about the process of bidding for works, services or equipment in a Public Administration such as the UCO, showing the transparency of the process. In the pilot experience of the previous academic course, the students positively valued attendance at a public act of opening economic offers of a real bidding process developed by the UCO, where they participated and showed how the whole transparent process rigorously follows the Law of Public Sector Contracts.

Key Words: engineering education, engineering project, entrepreneurship. 


\section{INTRODUCCIÓN}

Debido a la crisis económica de los últimos años, España está sufriendo una tasa de desempleo muy elevada. El ratio de desempleados en jóvenes menores de 25 años está en torno al 60\% (58,6\%) según el Ministerio de Empleo y Seguridad Social (Consultada junio 2019). Muchos de nuestros licenciados e investigadores tienen que emigrar a otros países por este motivo dando lugar a la denominada 'fuga de cerebros'. Ante esta situación la perspectiva actual es muy preocupante por lo que resulta esencial poner en práctica nuevas disciplinas y métodos de mejora de las habilidades empresariales y creativas en los estudiantes. En paralelo aparece también un incremento en la financiación por parte de las Universidades para la creación de spin-off o empresas de base tecnológica, por lo que desde el punto de vista académico resulta interesante conocer las políticas más efectivas para promoverlas, el seguimiento del proceso de empresarial desde su creación y las características personales del alumnado que decide crear su propia compañía (Abreu \& Grinevich 2013). Debido a estos motivos, el emprendimiento ha tenido un gran auge en estos últimos años dado el dinámico crecimiento y la natural evolución de la economía (Acs, Audretsch et al. 2012, Audretsch, Belitski et al. 2015, Memili, Fang et al. 2015) (ver figura 1)

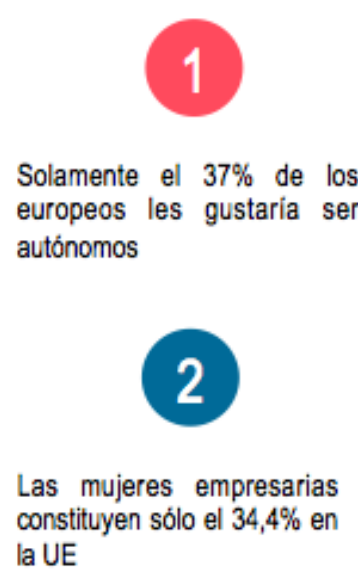

la UE
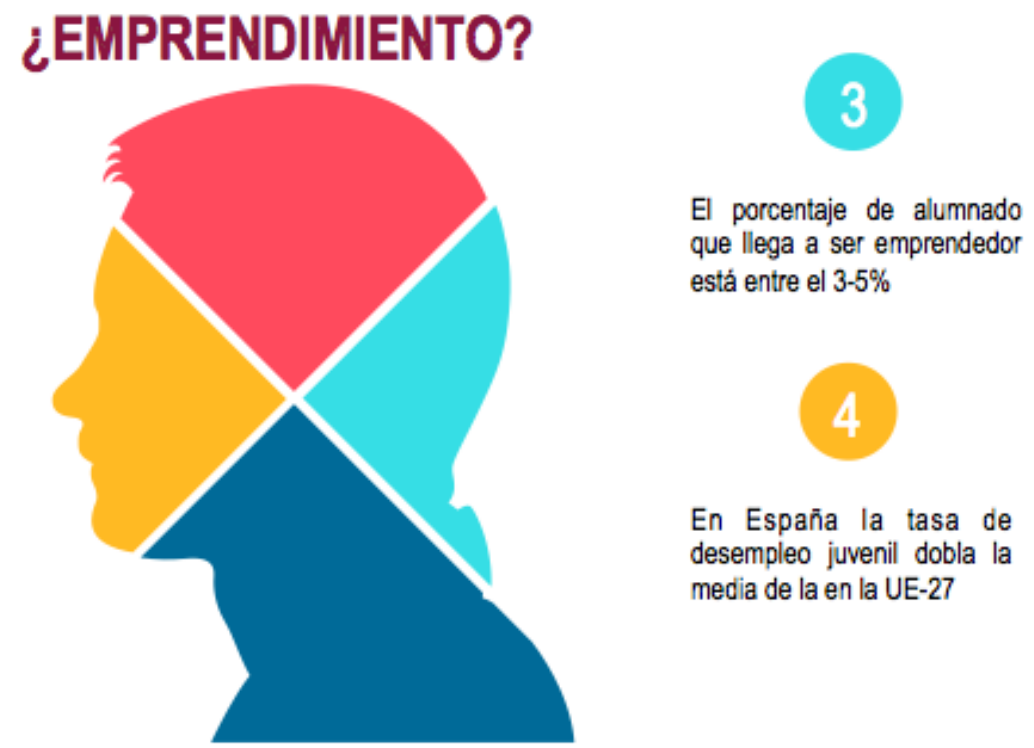

En España la tasa de desempleo juvenil dobla la media de la en la UE-27

Figura 1. Importancia del emprendimiento. Fuente: Elaboración propia.

A través de su enfoque en el desarrollo de nuevos productos y procesos (Morris, Neumeyer et al. 2015), el emprendimiento incrementa el número de mercados existentes y también aumenta el nivel de competitividad de los mismos (Rocha 2004). Esto, sin duda, beneficia tanto al consumidor como al empresario (Reynolds, Bosma et al. 2005). Los nuevos retos empresariales también activan las oportunidades de empleo para la gente joven (Thurik, Carree et al. 2008) y atraen a inversores extranjeros (Hermes and Lensink 2003). La importancia económica del emprendimiento está ampliamente descrita en la literatura (Landstrom, Harirchi et al. 2012). Como se ha comentado anteriormente, el emprendimiento se considera un propulsor del crecimiento económico y la creación de empleo (Shane 2000, Díaz-Casero, Ferreira et al. 2012, GEM, 2014). Esto contribuye a la formación bruta de capital fijo. Debido a estas consecuencias positivas sobre la economía, los responsables políticos de todo el mundo han comenzado a enfatizar el suministro de estructuras institucionales y de apoyo social para la creación de nuevos negocios (Minniti, 2008). Se está fomentando la innovación, 
se está favoreciendo la inversión y se están construyendo infraestructuras y marcos normativos que apuntan a promover el espíritu empresarial (Acs \& Szerb, 2007, Alhorr, Moore et al. 2008, Minniti, 2008).

En este contexto, un amplio número de programas y servicios, como por ejemplo, soporte en la creación de planes de negocio, centros educativos y cátedras de emprendimiento, se han creado para dar un mejor apoyo e infraestructuras a las nuevas empresas (Nab, Bulte et al. 2013). Parte de estos programas están dirigidos a los estudiantes universitarios como futuros emprendedores. Ya en el año 1998, la UNESCO señalaba en su "Declaración mundial sobre la educación superior en el siglo XXI: Visión y Acción" que la necesidad de aprender a emprender y fomentar el espíritu de iniciativa deben convertirse en importantes preocupaciones de la educación superior, a fin de facilitar las posibilidades de empleo de los egresados, que cada vez estarán más llamados a crear puestos de trabajo y no a limitarse a buscarlos. A principios de 2000 , la Organización para la Cooperación Económica y Desarrollo (OECD) recomienda que todos los países miembros deberían incluir asignaturas relacionadas con el emprendimiento en todos los niveles educativos. El informe Global Entrepreneurship Monitoring (GEM) elaborado por el Instituto de Empresa en 2010, señala que las actitudes de la sociedad española hacia el emprendimiento no difieren sustancialmente de las observadas para el conjunto de los países cuyas economías están conducidas por la innovación o de las del conjunto de países de la Unión Europea. Estos resultados cuestionan la idea tantas veces repetida de que la sociedad española no es emprendedora y ponen el foco de atención sobre otros aspectos que también inciden en la actividad emprendedora, como son las condiciones del entorno. Entre las condiciones del entorno peor valoradas por los expertos participantes en el informe están la insuficiente educación y formación específica en gestión emprendedora. Por esta razón, desde muchas instancias se están fomentando programas educativos para desarrollar la competencia de emprender, enfocada al emprendimiento económico o tecnológico, pero cuidando también el aspecto de iniciativa, capacidad de riesgos y elaboración de proyectos personales de vida. En Europa se marcan cuatro objetivos estratégicos para el 2020 en torno a la educación en emprendimiento (ver figura 2).

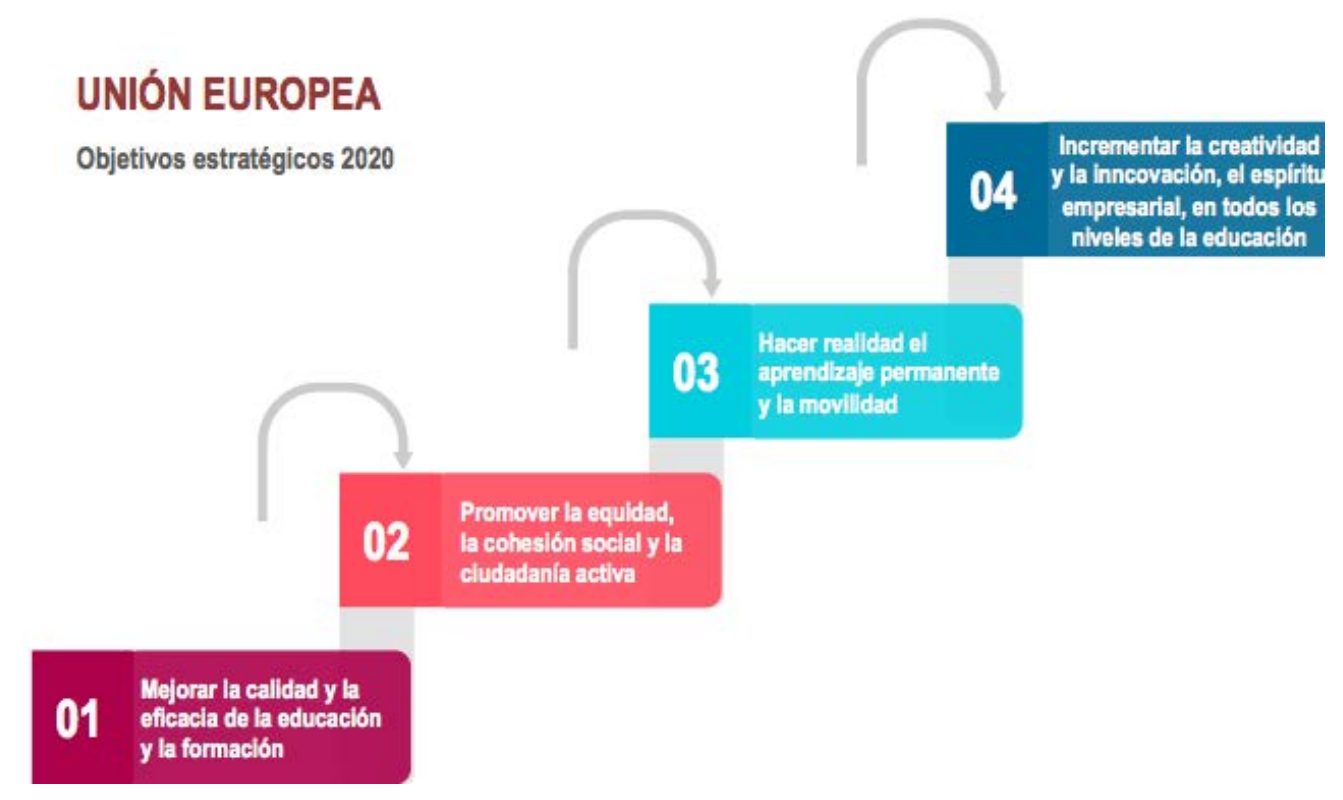

Figura 2. Objetivos estratégicos en la Unión Europea 2020.

Fuente: Elaboración propia. 
Para cumplir con estos objetivos la UE establece una serie de acciones a través del desarrollo de dos proyectos de investigación (http://ec.europa.eu/growth/smes/promoting-entrepreneurship_en):

Rethinking Education. Orientado a reformar los sistemas educativos en UE para satisfacer la creciente demanda de mayores niveles de cualificación (http://ec.europa.eu/education/policy/multilingualism/rethinking-education_en).

Entrepreneurship2020 Action Plan. Eduación y formación en emprendimiento. Eliminar las barreras administrativas. Reavivar la cultura del espíritu empresarial. (http://ec.europa.eu/growth/smes/promoting-entrepreneurship/action-plan/).

Muy pocos países en la UE disponen de políticas estatales de educación para el emprendimiento (EE) (ver figura 3).

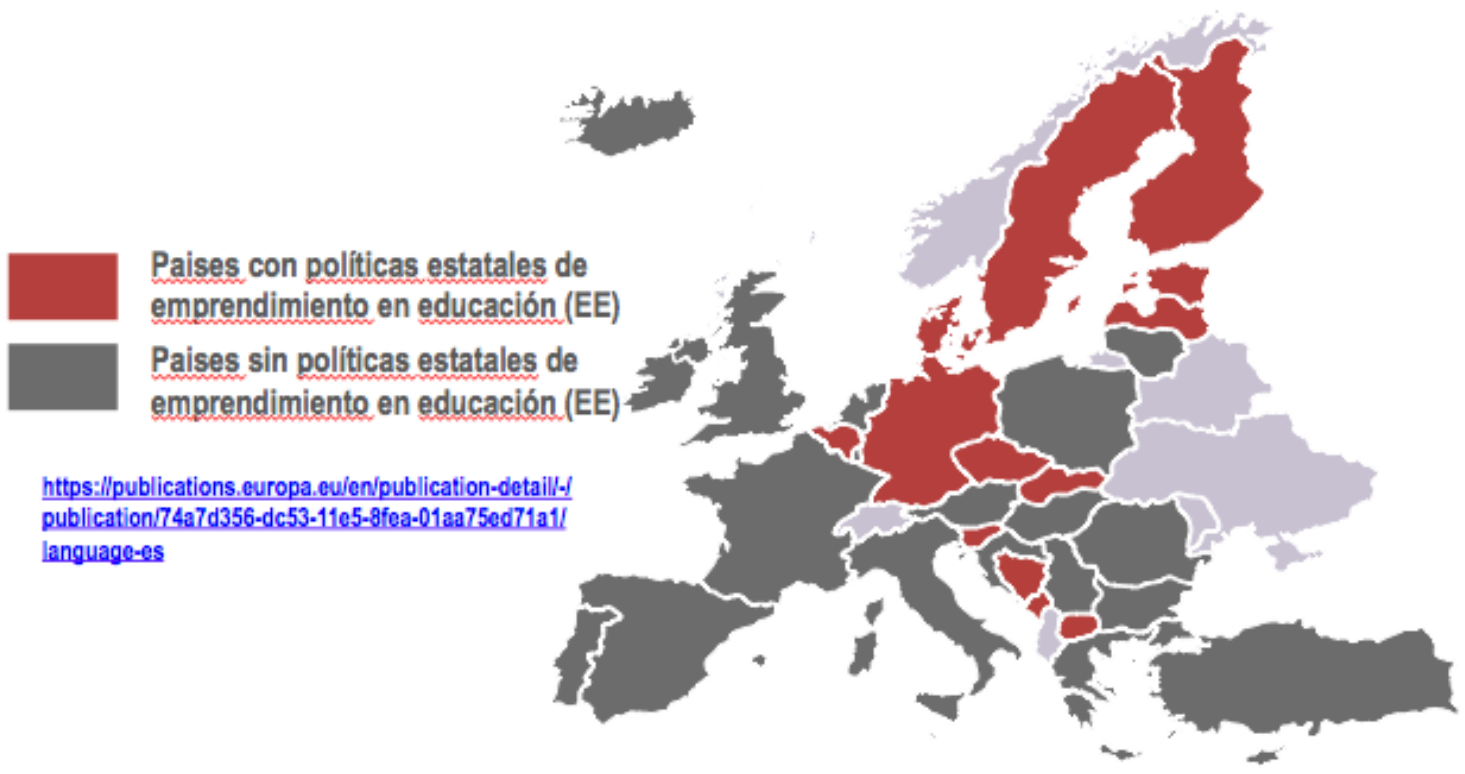

Figura 3. Países en Europa con EE.

Fuente: Elaboración propia.

En España, no hay una política estatal para educación para el emprendimiento, así, tales asignaturas han sido solo incluidas en el currículum de determinadas carreras universitarias, específicamente en el Grado de Ingeniería se oferta como Economía de la Empresa. En España, es a nivel autonómico donde se establece en muy pocas comunidades autónomas políticas específicas de EE. Sin embargo, a pesar del incremento de la atención y los esfuerzos por los países y las políticas globales, el ratio de creación empresarial se mantiene muy bajo (Liñán \& Fernandez-Serrano, 2014). La preferencia por el espíritu emprendedor entre los jóvenes universitarios no es muy alta (Nabi, Holden et al. 2010, van Praag 2011, GEM 2014), aunque los beneficios económicos de la educación formal para los empresarios sí que es elevada (van Praag, van Witteloostuijn et al. 2013). Poco a poco se está intentando fomentar el atractivo del emprendimiento a los estudiantes ya que esto supone una forma de introducirse en el mercado laboral sin perder su independencia (Martínez, Mora et al. 2007, Barros 2015). También se ha observado una creciente decepción en las ocupaciones tradicionales en las grandes empresas (Yemini \& Yeheskel 2011), en las que los procesos de reestructuración han implicado un recorte salarial y han erosionado las anteriores ventajas relacionadas con las ventajas de estar trabajando en una compañía como la estabilidad laboral, incentivos y seguridad (Astebro, Bazzazian et al. 2012).

Actualmente hay un gran número de investigaciones que sostienen la relevancia de las intenciones de emprendimiento entre los estudiantes universitarios en la decisión 
de empezar un nuevo negocio (Wu \& Wu 2008, Tolentino, Sedoglavich et al. 2014). Los estudios sobre las intenciones de emprendimiento con frecuencia descuidan la heterogeneidad de los antecedentes educacionales individuales. Entre los pocos estudios que incluyen en sus muestras diversidad de estudiantes o campos de estudio (17\%), la mayoría no analiza la potencialidad de la intención empresarial en relación al campo de estudio: estudios empresariales vs no empresariales (Wu \& Wu 2008, Rittippant, Kokchang et al. 2011); estudios tecnológicos vs no tecnológicos (Zhang, Duysters et al. 2014); estudios que no son de ciencias (ingeniería, empresariales o ciencias de la computación) vs los de ciencias naturales (Wang, Wong et al. 2002); estudios de humanidades y empresariales vs estudios de ciencias o tecnología (Schwarz, Wdowiak et al. 2009); estudios de administración de empresas, económicas y derecho, ciencias naturales e ingeniería y otros de ciencias sociales (Zellweger, Sieger et al. 2011). En este sentido, los resultados encuentran que en las carreras tecnológicas o de ingeniería muestra mayor predisposición por el emprendimiento. Por todos estos motivos, resulta interesante fomentar entre los estudiantes la capacidad para emprender, motivándolos y dándoles conceptos generales para el desarrollo de sus ideas de negocio.

La idea de abordar el emprendimiento desde la universidad tiene como propósito influir en los enfoques y comportamientos de los estudiantes de tal forma que muestren una mayor capacidad para resolver problemas con iniciativa y creatividad y desempeñen un papel más protagonista en la construcción de su propio proyecto de vida y, por consiguiente, actúen como verdaderos autores del cambio en los emprendimientos sociales, políticos, culturales y económicos, contribuyendo al desarrollo sostenible y sustentable de su comunidad. Hoy más que nunca necesitamos jóvenes emprendedores, con ideales y valores bien definidos, conscientes de la situación actual, y capaces de afrontar los retos del presente y del futuro y así comenzar a reducir aquellos estudiantes acomodados y sistematizados. Para ello, es importante el abordaje de la competencia emprendedora sin que ello suponga la inclusión de una nueva asignatura. Es más acertado pensar que es un principio transversal. A nivel universitario se evidencia un creciente interés por este tema que, si bien en un principio sólo era visible en carreras orientadas a la administración de empresas, ha comenzado a apreciarse en estudios de ingeniería.

Enseñar y aprender a emprender está directamente vinculada al desarrollo de proyectos de ingeniería. Con este proyecto docente se aborda el emprendimiento desde el punto de vista de que el proyecto de emprendimiento tiene como objetivo que los estudiantes logren identificar que, más allá de los conocimientos técnicos específicos, se demandará de ellos capacidad de trabajar en equipo, de identificar y solucionar problemas, de fijar objetivos y metas, de buscar y analizar información, de negociar, de generar redes de apoyo, de liderar, y de comunicarse, entre otras. La formación de emprendedores basada en proyectos trata de replicar el mundo real de los negocios al mismo tiempo que permite el desarrollo educativo. El modelo busca formar personas altamente capaces, con una clara conciencia del entorno económico y social en el que se desenvuelven, con especial énfasis en el desarrollo de su espíritu y habilidades de emprendedores creativos, con un enfoque hacia la generación de ideas innovadoras y con un alto grado de tecnología.

De lo anterior se desprende que la formación de emprendedores en la asignatura de Proyectos implica la ampliación de los límites que clásicamente impone el espacio del aula a partir de la integración con el contexto: el alumnado, a medida que desarrollan un proyecto, deben tomar contacto con el entorno, incluyendo, por ejemplo, potenciales clientes y proveedores reales, y analizar la viabilidad del mismo. 
El objetivo principal es fomentar el emprendimiento entre los estudiantes de cuarto del Grado de Ingeniería Eléctrica. Durante el curso académico 2016/17, se obtuvieron resultados muy positivos, analizándose la intención empresarial de los estudiantes antes y después de cursar la asignatura. Más de un $90 \%$ de los estudiantes cambiaron de opinión y su intención de desarrollar su idea de negocio al finalizar la asignatura. Hubo dos desarrollos de ideas para patentes y dos estudiantes presentaron su idea de negocio al Concurso Yuzz de la Cátedra Santander ganando el primer premio consistente en un viaje a Silicon Valley y dando acceso al concurso a nivel nacional del Banco Santander. Además, se pretende realizar una segunda experiencia docente del proyecto donde el alumnado participante durante el curso académico 2017/18 participen de 'Coach' para el alumnado de otras asignaturas transversales e incluso para la asignatura de Proyectos del curso 2018/19.

\section{DESARROLLO DE LA EXPERIENCIA DE INNOVACIÓN}

\subsection{Creación de empresas}

La primera fase de actividad académicamente dirigida consiste en instar a que el alumnado forme 'empresas', grupos de cinco o seis estudiantes, a la que tendrán que dar un nombre y establecer un organigrama designando a un director general, un director comercial, un director financiero y un director técnico del que dependerán dos empleados (ver figura 4).

Cada uno de estos 'actores' tienen una función: el director general será el responsable máximo del éxito del trabajo llevado a cabo realizando una distribución temporal, reparto de la carga de trabajo y que hará para cada trabajo realizado. El director financiero tiene la responsabilidad de decidir cuestiones económicas que se plantean en el desarrollo de los trabajos. El director comercial será el responsable de vender el trabajo realizado a los 'inversores', mediante el concepto de 'startup', haciendo atractiva la idea propuesta, planteándose previamente las cuestiones que se harían los inversores. El director técnico junto con su equipo de trabajo, y en coordinación con el resto de miembros de la empresa, harán varias propuestas alternativas, estudiarán la viabilidad técnica y la económica y se decidirá la mejor idea propuesta y se desarrollará. Los trabajadores serán los responsables de llevar a cabo el proyecto técnico bajo la supervisión del director técnico. La primera fase de actividad académicamente dirigida consiste en instar a que el alumnado forme 'empresas', grupos de cinco o seis estudiantes, a la que tendrán que dar un nombre y establecer un organigrama designando a un director general, un director comercial, un director financiero y un director técnico del que dependerán dos empleados (ver figura 4).

Cada uno de estos 'actores' tienen una función: el director general será el responsable máximo del éxito del trabajo llevado a cabo realizando una distribución temporal, reparto de la carga de trabajo y que hará para cada trabajo realizado. El director financiero tiene la responsabilidad de decidir cuestiones económicas que se plantean en el desarrollo de los trabajos. El director comercial será el responsable de vender el trabajo realizado a los 'inversores', mediante el concepto de 'startup', haciendo atractiva la idea propuesta, planteándose previamente las cuestiones que se harían los inversores. El director técnico junto con su equipo de trabajo, y en coordinación con el resto de miembros de la empresa, harán varias propuestas alternativas, estudiarán la viabilidad técnica y la económica y se decidirá la mejor idea propuesta y se desarrollará. Los trabajadores serán los responsables de llevar a cabo el proyecto técnico bajo la supervisión del director técnico. 


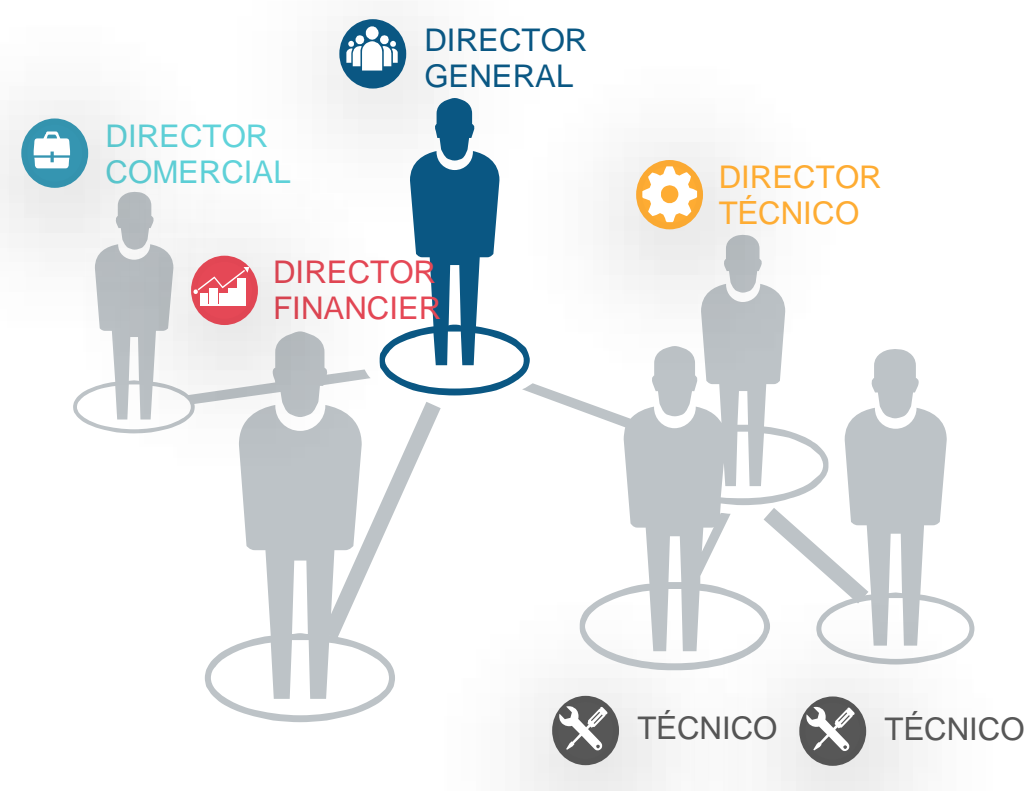

Figura 4. Organigrama de la empresa.

Fuente: Elaboración propia.

¿Quiénes son los 'inversores'? Son el profesorado, ya que irán concediendo crédito para el desarrollo de los proyectos empresariales. El sistema de créditos, las bonificaciones y las actividades implicadas se especificarán mediante un contrato.

\subsection{Contexto teórico del proyecto propuesto}

Este proyecto docente se lleva a cabo dentro de la asignatura de Proyectos del Grado de Ingeniería Eléctrica, aunque la metodología llevada a cabo es totalmente extensible a otras asignaturas, al tratarse de trabajo de competencias transversales. La asignatura de Proyectos tiene un total de 60 horas, siendo 36 horas para grupo grande y 24 para grupos medianos. Las 36 horas para grupo grande se dividen en tres grandes bloques:

- Bloque I. Teoría de Proyectos.

- Bloque II. Planificación, programación y control de Proyectos.

Dentro del Bloque I se realizan una serie de actividades a desarrollar por 'contrato'. La primera Sesión Introductoria de la asignatura se lleva a cabo durante hora y media, donde se les presenta la asignatura, la metodología, leyendo la guía docente de la misma. Además se les pasa un test inicial sobre sus perspectivas laborales cuando finalicen sus estudios. Es en esta primera sesión introductoria donde el alumnado tiene que formar las empresas, indicando un nombre para la misma junto con los miembros que la formarán y sus responsabilidades.

\subsection{El contrato}

Una vez establecidas las responsabilidades, se firma un contrato entre las empresas y los inversores donde se establecen los trabajos a realizar, las responsabilidades de 
ambos y el pago por cada uno de los trabajos. En la figura 5 se puede ver el contrato legal y vinculante.

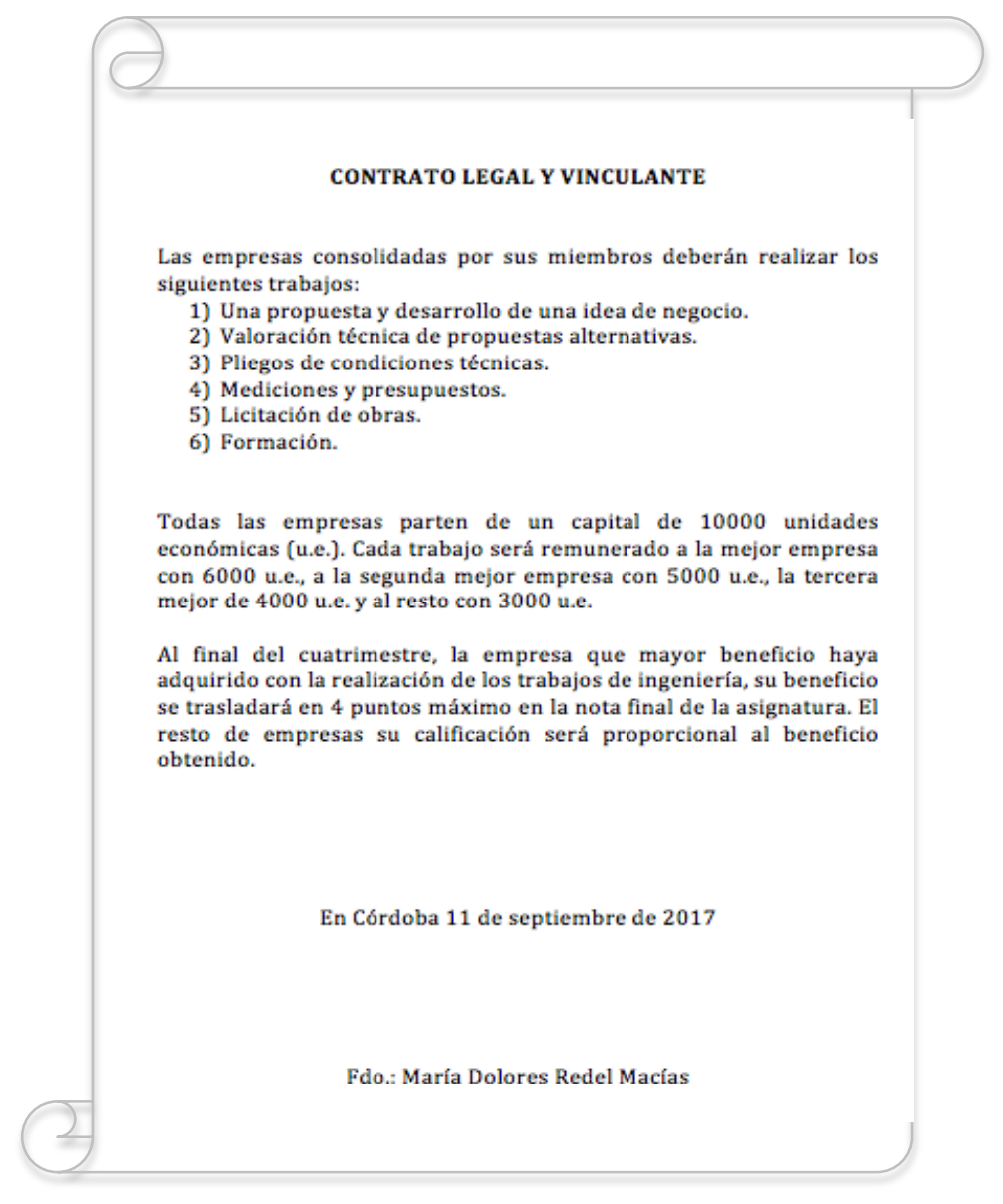

Figura 5. Contrato legal y vinculante. Fuente: Elaboración propia.

\section{RESULTADOS OBTENIDOS Y DISCUSIÓN}

Los trabajos a desarrollar por las empresas están especificados en la figura 6. Cada trabajo será valorado económicamente por 6000 unidades económicas (u.e.) y cada empresa parte de un capital inicial de 10000 u.e. La empresa que presente el mejor trabajo tendrá una remuneración de 6000 u.e., la segunda mejor de 5000 u.e., la tercera mejor de 4000 u.e., teniendo el resto una valoración de su trabajo de 3000 u.e. Este capital será el que los inversores les irán dando para la financiación de su proyecto empresarial y desarrollo de su idea de negocio. Por tanto, la empresa que más beneficio económico tenga debido al desarrollo de sus trabajos, necesitará de menor financiación externa o apoyo de inversores para el desarrollo de la misma.

Por otro lado, durante el desarrollo de las sesiones teóricas se propone al alumnado un incentivo extra para ganar capital. Mediante un sistema de test online en las presentaciones teóricas, utilizando un sistema de mandos a distancia, las empresas tienen la oportunidad de valorar los conocimientos adquiridos a lo largo del tema y de la asignatura ya que se van intercalando preguntas del tema actual y de otros anteriores. Con el sistema de mandos responden al tipo test entre varias opciones de respuesta, dejando 20s o menos, en función de la dinámica de la clase, para contestar. Al finalizar el tema se comprueban las puntuaciones adquiridas por las distintas empresas, teniendo el privilegio la/s empresa/s que mayor puntuación haya conseguido de 'robar' capital a la empresa que decidan (1000 u.e.). De este modo, el director de la empresa fomenta y 
alienta al resto de empleados sobre la importancia de asistir a clase para tener mayor soporte a la hora de la realización del test. El gerente de la empresa será el encargado de llevar al día el estado de capital de su empresa y del resto, así como de decidir con esta información a que empresa le realiza el 'robo' de capital. Al final del cuatrimestre, el capital obtenido por cada empresa se convierte en puntos extra de calificación de la asignatura. La empresa con mayor beneficio económico tendrá 1,5 puntos adicionales en la calificación final, y el resto de empresas se calcula de forma proporcional. Al finalizar el semestre, el alumnado realiza otra encuesta sobre sus perspectivas laborales para comparar con el test inicial llevado a cabo. La figura 7 muestra un esquema de los pasos en la metodología docente.

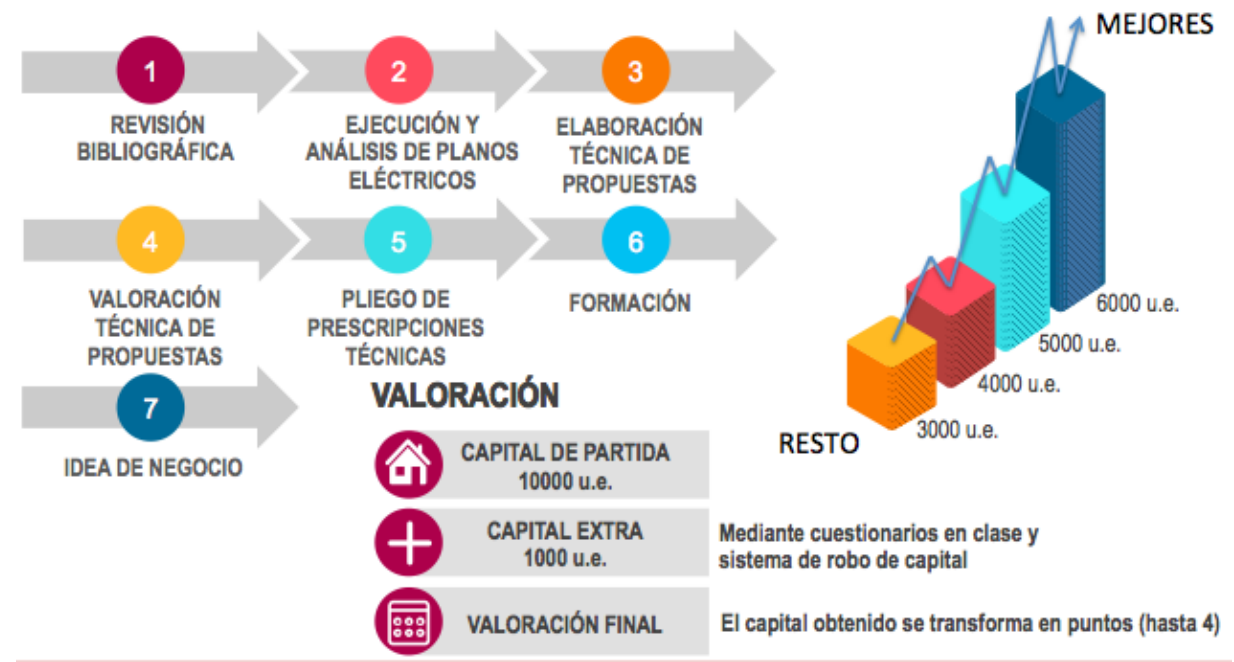

Figura 6. Sistema de valoración de actividades. Fuente: Elaboración propia.
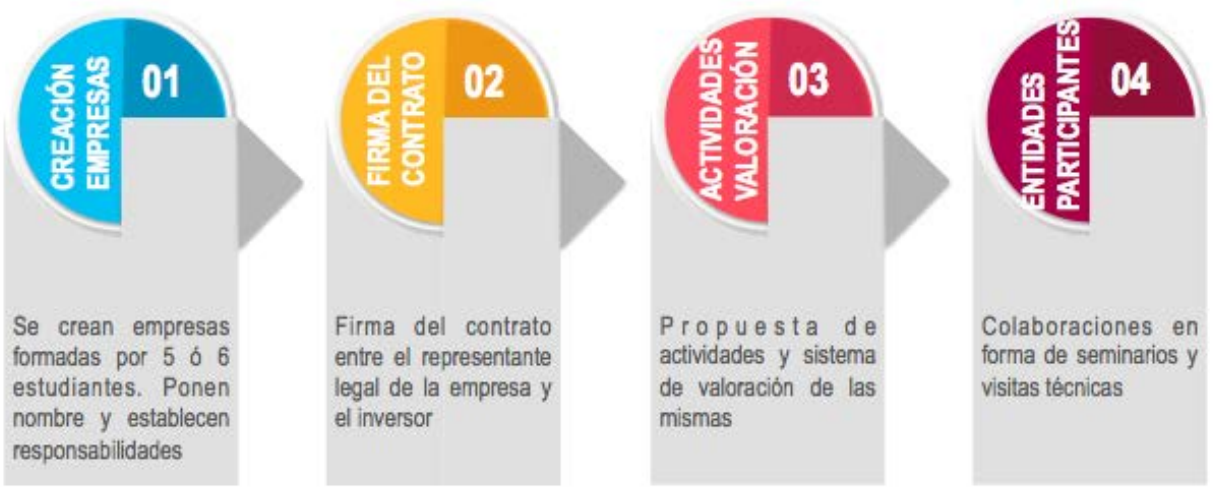

Para el desarrollo de los trabajos se cuenta con la colaboración del Vicerrectorado de Coordinación Institucional e Infraestructuras de la Universidad de Córdoba (UCO), la Jefa del Servicio de Contratación y Patrimonio (UCO), y de la Asesoría Jurídica (UCO), la Cátedra Santander de Emprendimiento (UCO), el vivero de empresas municipal de Córdoba y el Colegio de Ingenieros Técnicos Industriales de Córdoba.

Los trabajos propuestos se desarrollan a continuación: 
I. Propuesta de idea de negocio y defensa. Las empresas a lo largo del cuatrimestre y con los conocimientos adquiridos en el mismo, tienen que desarrollar una idea de negocio. La defensa de la misma se lleva a cabo la última semana de clase. Para ello se dispone de un tribunal evaluador externo formado por la Jefa del Servicio de Contratación y Patrimonio, el Vicerrector de Coordinación Institucional e Infraestructuras y un miembro de la Asesoría Jurídica. La mejor idea y exposición de la misma es premiada con una Tablet, la segunda mejor idea con un disco duro portátil y la tercera mejor idea con una memoria USB.

II. Valoración de juicio de valor de propuestas. En coordinación con el Vicerrectorado de Infraestructuras, se proporcionan ofertas técnicas y económicas (anónimas) para su valoración por el alumnado considerando diversos criterios, tal y como se hace para la adjudicación de concursos públicos. Para ello se consulta la plataforma de contratación del Estado que se utiliza en la UCO para sus concursos públicos: https://contrataciondelestado.es/wps/portal/!ut/p/b0/DccxCoAwDADAJ8VZcFB3 wck2W6BRgmkaS_D9etsBQglOeuWikGak_3NhdhW7R-diq7NolOQBcMBCChlcchdM9PT9iONE3it8wdzn_H7/.

III. Elaboración de un pliego de condiciones. En base a algún proceso abierto que tengan en el Servicio de Contratación y Patrimonio (obra, servicio o equipamiento), las empresas debe de realizar un pliego de condiciones para su concurso (ficticio).

IV. Mediciones y presupuesto. Siguiendo el mismo proceso, se elige un concurso público y las empresas deberán realizar su oferta económica para el mismo.

V. Formación. Se realizan seminarios estructurados en base a los objetivos e intereses para las empresas. Un primer seminario impartido por personal de la Cátedra Santander Emprendimiento, para dar información sobre la elaboración de planes de negocio, etapas de implantación de una empresa y la plataforma de lanzamiento de ideas de la Cátedra Santander. El segundo seminario es impartido por la Jefa de Contratación y Patrimonio o el Vicerrector de Coordinación Institucional e Infraestructuras sobre el sistema de contratación público de obras. El tercer seminario lo imparte el Secretario del Colegio Oficial de Ingenieros Técnicos de Córdoba. Finalmente, el alumnado, con el permiso del Vicerrector de Coordinación Institucional e Infraestructuras puede asistir al acto público de apertura de sobres número 3 de un proceso de licitación abierto que tenga la UCO, si es posible (ver figura 8).

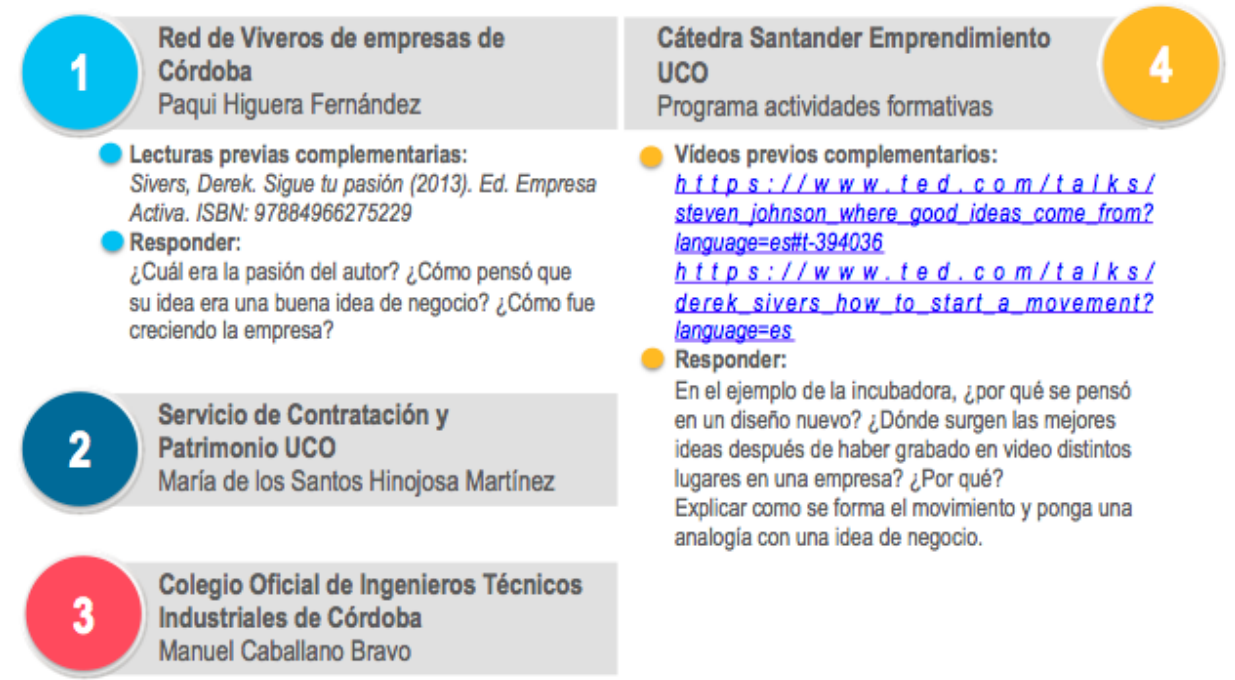

Figura 8. Seminarios impartidos.

Fuente: Elaboración propia. 
Con este proyecto docente se ha pretendido dar una visión o perspectiva al alumnado desde el punto de vista de la creación del autoempleo. Según el diario el Mundo, por primera vez los estudiantes universitarios españoles que tienen pensado crear un negocio $(26,8 \%)$ supera a los que se ven trabajando en la Administración Pública el (25\%), ese fue el resultados de las 8600 encuestas realizadas por GAD3 para el estudio encargado por Educa 2020, donde Ingeniería y Arquitectura son las ramas con mayores porcentajes de alumnado que quieren crear una empresa. Además según la UE, cuando el alumnado forma parte de un programa de EE se pasa de tener un porcentaje de alumnado emprendedor del 3-5\% al 15-30\%. De este modo se cumpliría mediante este proyecto con los objetivos marcados por la UE. Con estos datos, se pretende trabajar en esta línea para estimular al alumnado en la generación de ideas de negocio y dar soporte para llevarlas a cabo. Por otro lado, a través del trabajo coordinado con el Vicerrectorado de Coordinación Institucional e Infraestructuras se intenta acercar al alumnado cómo es el proceso de licitación de obras, servicios o equipamiento en una Administración Pública como es la UCO, mostrando la transparencia del proceso. En la experiencia piloto del anterior curso académico 2016/2017, el alumnado valoró muy positivamente la asistencia a un acto público de apertura de ofertas económicas de un proceso de licitación real desarrollado por la UCO, donde se mostraron participativos y vieron de primera mano cómo todo el proceso es transparente y sigue rigurosamente la Ley de Contratos del Sector Público.

La experiencia durante el curso académico 2016/2017 fue muy positiva, valorando el alumnado muy positivamente la experiencia llevada a cabo mediante un test de satisfacción. En este test se preguntaron cuestiones tales como: ¿Montaría usted su propio negocio?; así como otras cuestiones relativas a la satisfacción con la metodología llevada a cabo y con los conceptos clave de la asignatura. Así mismo, hubo dos alumnos que presentaron su idea de negocio al programa Yuzz Jóvenes con Ideas ganando el primer premio. En las figuras 9 y 10 se muestran un díptico realizado por el alumnado como propaganda de su empresa y las imágenes de un power point de la idea de negocio presentada por los ganadores del concurso Yuzz Jóvenes con Ideas.
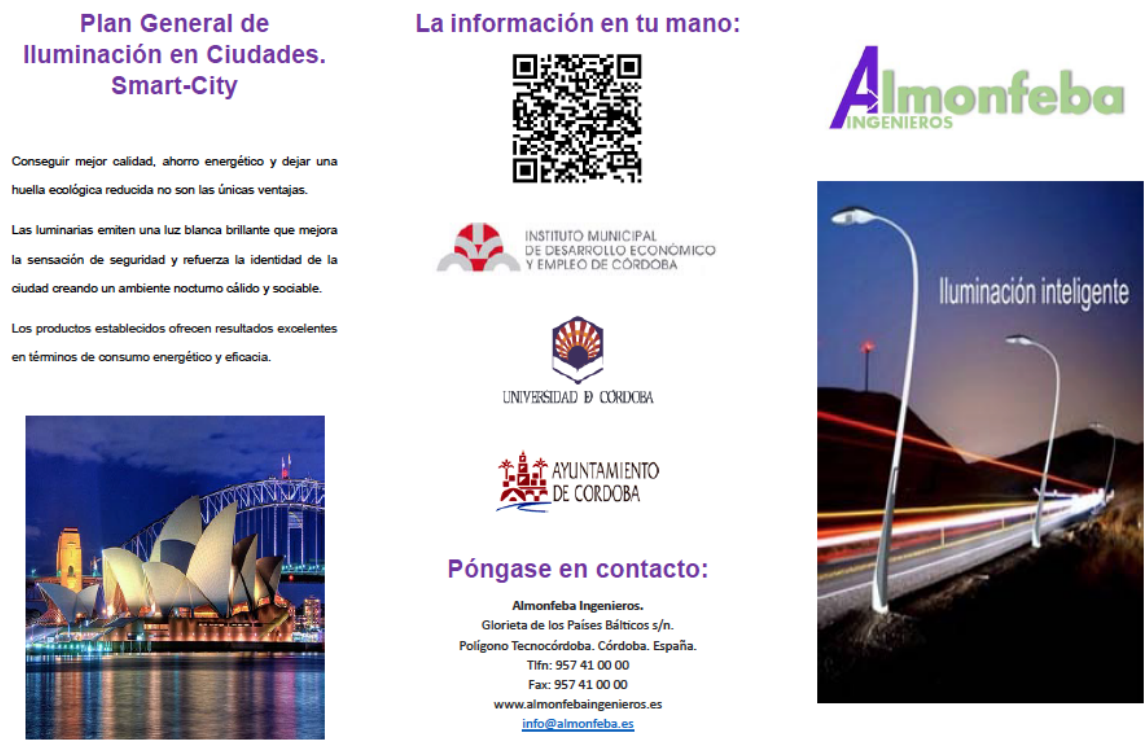

Figura 9. Díptico de la idea de negocio de la empresa Almonfeba Ingenieros.

Fuente: Elaboración propia. 


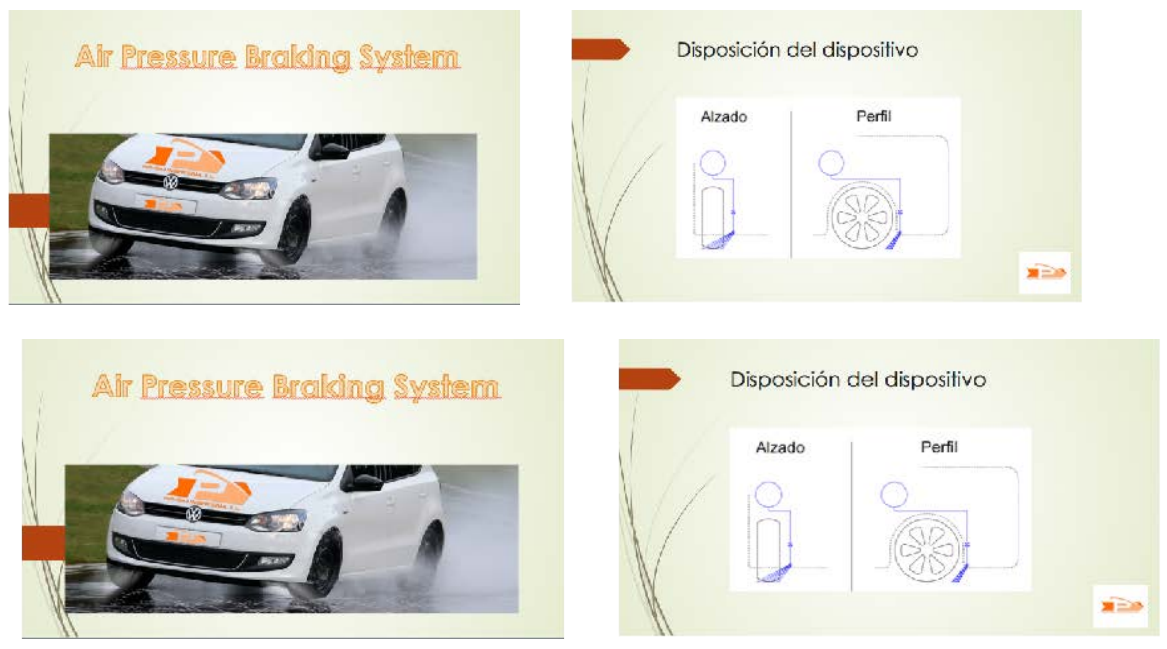

Figura 10. Vídeo de la idea de negocio de la empresa Posadas Ingeniería S.L. Fuente: Elaboración propia.

\section{CONCLUSIONES}

Las conclusiones a las que se han llegado quedan resumidas a continuación.

- En un test final de satisfacción realizado al alumnado, comparado con los resultados del test inicial, un $90,0 \%$ del alumnado montaría su propio negocio.

- Los resultados académicos han mejorado con respecto a otros años.

- En el test final, el 85,9\% del alumnado contestó tener claro lo que era un proyecto de ingeniería y su estructura documental.

- En el test final, el 95,7\% del alumnado contestó tener claro cómo realizar la bibliografía, un presupuesto y un pliego

- Se ha fomentado capacidades de dirección y gestión de proyectos entre otras capacidades IPMA.

- Valoración muy positiva del alumnado del proyecto docente llevado a cabo (4,8 en escala 1-5).

\section{REFERENCIAS}

Ministerio de empleo y seguridad social. Gobierno de España. Recuperado de http://www.empleo.gob.es/es/estadisticas/.

Abreu, M. \& Grinevich, V. (2013). The nature of academic entrepreneurship in theUK: Widening the focus on entrepreneurial activities. Research Policy 42(2), 408-422.

Acs, Z. J., D. B. Audretsch, P. Braunerhjelm \& B. Carlsson (2012). Growth and entrepreneurship. Small Business Economics 39(2), 289-300.

Acs, Z. J. \& Szerb, L. (2007). Entrepreneurship, economic growth and public policy. Small Business Economics 28(2-3), 109-122.

Alhorr, H. S., Moore, C.B. \& Payne, G.T. (2008). The impact of economic integration on cross-border venture capital investments: evidence from the European Union. Entrepreneurship Theory and Practice, 32(5), 897-917. 
Astebro, T., Bazzazian, N. \& Braguinsky, S. (2012). Startups by recent university graduates and their faculty: implications for university entrepreneurship policy. Res Policy 41(4), 663-677.

Audretsch, D. B., Belitski, M. \& Desai, S. (2015). Entrepreneurship and economic development in cities. The Annals of Regional Science 55(1), 33-60.

Barros, A. (2015). Crisis, what crisis? The life goals of university-aged students during an ongoing economic and financial crisis. Current Psychology 34, 434-446.

Díaz-Casero, J., Ferreira, J., Mogollón, R. \& Raposo, M. (2012). Influence of institutional environment on entrepreneurial intention: a comparative study of two countries university students. International Entrepreneurship Management Journal 8(1), 5574.

GEM (2014). Global entrepreneurship monitor 2014 global report.

Hermes, N. \& Lensink, R. (2003). Foreign direct investment, financial development and economic growth. The Journal of Development Studies, 40(1), 142-163.

Landstrom, H., Harirchi, G. \& Astrom, F. (2012). Entrepreneurship: exploring the knowledge base. Res Policy 41(7), 1154-1181.

Liñán, F. \& Fernandez-Serrano, J. (2014). National culture, entrepreneurship and economic development: different patterns across the European Union. Small Business Economics 42(4), 685-701.

Martínez, D., Mora, J.-G. \& Vila, L. (2007). Entrepreneurs, the self-employed and employees amongst young European higher education graduates. Eur J Educ 42(1), 99-117.

Memili, E., Fang, H., Chrisman, J.J. \& DeMassis, A. (2015). The impact of smallandmedium-sized family firms on economic growth. Small Business Economics 45(4), 771-785.

Minniti, M. (2008). The role of government policy on entrepreneurial activity: productive, unproductive, or destructive? Entrepreneurship. Theory and Practice 32(5), 779790.

Morris, M., Neumeyer, X. \& Kuratko, D. F. (2015). A portfolio perspective on entrepreneurship and economic development. Small Business Economics 45(4), 713-728.

Nab, J., Bulte, A. \& Pilot, A. (2013). Fostering the competence of science students in identifying business opportunities: a design research approach. Int $\mathrm{J}$ Entrep Ventur $5(1), 28-47$.

Nabi, G., Holden, R. \& Walmsley, A. (2010). Entrepreneurial intentions among students: towards a re-focused research agenda. J Small Bus Enterp Dev 17(4), 537-551.

Reynolds, P., Bosma, N., Autio, E., Hunt, S., De Bono, N. \& Servais, I. (2005). Global entrepreneurship monitor: data collection design and implementation 1998-2003. Small Business Economics 24(3), 205-231.

Rittippant, N., Kokchang, W., Vanichkitpisan, P. \& Chompoodang, S. (2011). Measure of entrepreneurial intention of young adults in Thailand. Proceedings of the 
International Conference on Engineering, Project, and Production Management (EPPM 2011), Singapore.

Rocha, H. O. (2004). Entrepreneurship and development: the role of clusters. Small Business Economics 23(5), 363-400.

Schwarz, E., Wdowiak, M., Almer-Jarz, D. \& Breitenecker, R. (2009). The effects of attitudes and perceived environment conditions on students' entrepreneurial intent: an Austrian perspective. Educ Train 51(4), 271-291.

Shane, S. (2000). Prior knowledge and the discovery of entrepreneurial opportunities. Organ Sci 11(4),448-469.

Thurik, A. R., Carree, M. A., Van Stel, A. \& Audretsch, D. B. (2008). Does selfemployment reduce unemployment? Journal of Business Venturing 23(6), 673-686.

Tolentino, L., Sedoglavich, V., Lu, V. Garcia, P. \& Restubog, S. (2014). The role of career adaptability in predicting entrepreneurial intentions: a moderated mediation model. J Vocat Behav 85(3),403-412.

Van Praag, M. (2011). Who values the status of the entrepreneur? Handbook of research on innovation and entrepreneurship. D. Audretsch, O. Falck and S. Heblich. Edward Elgar, Cheltenham, 24-44.

Van Praag, M., Van Witteloostuijn, A. \& Van der Sluis, J. (2013). The higher returns to formal education for entrepreneurs versus employees. Small Bus Econ 40(2), 375396.

Wang, C., Wong, P. \& Lu, Q. (2002). Entrepreneurial intentions and tertiary education. Information Age Publishing, Greenwich, 55-82.

Wu, S. \& Wu, L. (2008). The impact of higher education on entrepreneurial intentions of university students in China. J Small Bus Enterp Dev 15, 752-774.

Yemini, M. \& Yeheskel, O. (2011). Not born here-evaluation of the country of origin effect on the entrepreneurial intentions of Israeli engineering students. Int J Learn 17(10), 329-342.

Zellweger, T., Sieger, P. \& Halter, F. (2011). Should I stay or should I go? Career choice intentions of students with family business background. J Bus Ventur 26(5), 521536.

Zhang, Y., Duysters, G. \& Cloodt, M. (2014). The role of entrepreneurship education as a predictor of university students' entrepreneurial intention. Int Entrep Manag J 10, 623-641. 